\title{
Serum Profiling Identifies Novel Muscle miRNA and Cardiomyopathy-Related miRNA Biomarkers in Golden Retriever Muscular Dystrophy Dogs and Duchenne Muscular Dystrophy Patients
}

Laurence Jeanson-Leh, * Julie Lameth, * Soraya Krimi, ${ }^{*}$ Julien Buisset, * Fatima Amor, ${ }^{*}$ Caroline Le Guiner, ${ }^{\dagger}$ Inès Barthélémy, Laurent Servais, ${ }^{\S}$ Stéphane Blot, ${ }^{\ddagger}$ Thomas Voit, ${ }^{\top \|}$ and David Israeli*

From Genethon, * Evry, France; INSERM UMR 1089, ${ }^{\dagger}$ Atlantic Gene Therapies, Nantes University, Nantes Hospital (Université de Nantes, CHU de Nantes), Nantes, France; the Neurobiology Unit (UPR de Neurobiologie), ${ }^{\ddagger}$ East Paris University (Université Paris-Est), National Veterinary School of Alfort (Ecole Nationale Vétérinaire d'Alfort), Maisons-Alfort, France; the Department of Therapeutic Trials and Databases, ${ }^{\S}$ Pierre and Marie Curie University (Université Pierre et Marie Curie) - Paris" UM 76, CNRS, UMR 7215, Myology Institute (Institut de Myologie), Paris, France; and INSERM, UMRS 974, ${ }^{\top}$ Paris, France

Accepted for publication

July 8, 2014.

Address correspondence to Laurence Jeanson-Leh, Ph.D., GENETHON, 1 rue de l'Internationale, 91002, Evry, France. E-mail: jeanson@ genethon.fr.

\begin{abstract}
Duchenne muscular dystrophy (DMD) is a fatal, X-linked neuromuscular disease that affects 1 boy in 3500 to 5000 boys. The golden retriever muscular dystrophy dog is the best clinically relevant DMD animal model. Here, we used a high-thoughput miRNA sequencing screening for identification of candidate serum miRNA biomarkers in golden retriever muscular dystrophy dogs. We confirmed the dysregulation of the previously described muscle miRNAs, miR-1, miR-133, miR-206, and miR-378, and identified a new candidate muscle miRNA, miR-95. We identified two other classes of dysregulated serum miRNAs in muscular dystrophy: miRNAs belonging to the largest known miRNA cluster that resides in the imprinting DLK1-DIO3 genomic region and miRNAs associated with cardiac disease, including miR-208a, miR-208b, and miR-499. No simple correlation was identified between serum levels of cardiac miRNAs and cardiac functional parameters in golden retriever muscular dystrophy dogs. Finally, we confirmed a dysregulation of miR-95, miR-208a, miR-208b, miR-499, and miR-539 in a small cohort of DMD patients. Given the interspecies conservation of miRNAs and preliminary data in DMD patients, these newly identified dysregulated miRNAs are strong candidate biomarkers for DMD patients. (Am J Pathol 2014, 184: 2885-2898; http://dx.doi.org/10.1016/j.ajpath.2014.07.021)
\end{abstract}

Duchenne muscular dystrophy (DMD) is an X-linked neuromuscular disease that affects 1 boy in 3500 to 5000 boys. This recessive lethal disorder is the most frequent form of muscular dystrophy in children. DMD is caused by a deficiency of the dystrophin protein that leads to progressive degeneration of skeletal and cardiac muscle tissues that is eventually replaced by fat and connective tissues. Death is provoked almost exclusively by respiratory or cardiac failure, with a growing proportion of cardiac failure in recent years. ${ }^{1,2}$ The best clinically relevant animal model for DMD is the golden retriever muscular dystrophy (GRMD) dog. ${ }^{3}$ The GRMD dog has a mutation in the sixth intron of the canine dystrophin gene, causing an aberrant splicing event, resulting in a premature stop codon and the absence of dystrophin protein. Clinically, symptoms in dogs are close to those in DMD patients: abnormal posture, locomotion defects, progressive muscle degeneration, fibrosis, adipogenesis, respiratory insufficiency, and dilated cardiomyopathy. ${ }^{4}$ Moreover, the GRMD model size is comparable with that of young DMD boys, providing an attractive advantage when translating treatments from animals to patients.

Classically, analytical methods for diagnosis and monitoring of DMD patients are based on muscle histology,

Supported by the Association Francaise contre les Myopathies (AFM) and Agence Nationale de la Recherche binational grant ANR-BMBF-09GENO-107 (T.V.). This work is a contribution to ADNA (Advanced Diagnostics for New Therapeutic Approaches), a program dedicated to personalized medicine, coordinated by Institut Mérieux and supported and partially funded by the French public agency, OSEO.

Disclosures: None declared. 
quantification of serum creatine kinase (CK), electromyography, electrocardiography, and DNA mutation analysis. Disease monitoring from muscle biopsies provides important indications but remains an invasive medical act that provides variable results, depending on the biopsy sites and the timing of sampling. The muscle isoform of CK is expressed in skeletal muscle and released into the circulation after muscle fiber membrane leakiness and/or breakdown. However, CK lacks specificity because it also rises after exercise. ${ }^{6}$ Moreover, in the GRMD model, the serum CK level might be affected by immunosuppressive treatments irrespective of therapeutic benefit and muscle phenotype,${ }^{7,8}$ further limiting its utility.

Several recent phase 2 and 3 clinical trials in DMD patients were aimed at the restoration of dystrophin expression. The evaluations of results in these clinical trials were based principally on the quantification of dystrophin expression in muscle biopsies and on a functional assessment of muscle capacity by using the 6-minute walk test. However, dystrophin expression varies, depending on the different muscles and biopsies, its correct quantification is technically not completely resolved, and its correlation to clinical benefit has not been established. Moreover, the measured performance of DMD boys in the 6-minute walk test does not depend merely on muscle capacity, and interpreting results is therefore another unresolved issue. ${ }^{9-12}$

Cardiac troponin (Tpn) is the gold standard serum biomarker for acute coronary syndrome and heart failure, although its elevation can also be associated with other conditions such as respiratory diseases or infections. ${ }^{13}$ Cardiac Tpn was found to correlate with cardiac pathology in DMD patients, ${ }^{14}$ which, however, could not be confirmed in another study. ${ }^{15}$ The N-terminal pro-brain natriuretic peptide, a hormone produced mainly by the cardiomyocyte of the ventricular wall, has also been evaluated previously as a biomarker in DMD patients. Ergul et al ${ }^{16}$ have identified significant elevated levels of both cardiac Tpn and N-terminal pro-brain natriuretic peptide in the serum of DMD patients with reduced left ventricular ejection fraction $(<55 \%)$ and increased risk of development of dilated cardiomyopathy. ${ }^{16}$ They therefore suggested the routine quantification of these two enzymes along with ECG/echocardiographic tests in DMD patients. In contrast, according to Schade van Westrum et al, ${ }^{17}$ the measurement of N-terminal pro-brain natriuretic peptide was not helpful for the diagnosis of dilated cardiomyopathy in DMD patients in any phase of the disease. These contradictory reports indicate the urgent necessity in the identification of reliable cardiomyopathy biomarkers for diagnosis, prognosis, and monitoring in DMD patients.

miRNAs are small-sized RNA molecules involved in post-transcriptional control of gene expression. Although these molecules are normally present and function inside cells, it was recently discovered that they are also secreted and can be detected in every body fluid. miRNAs are particularly stable in blood samples, and their abundance varies in correlation to the pathophysiological state of the tissue(s) of origin. ${ }^{18-20}$ Thus, serum miRNAs have the potential to be used as specific and reliable biomarkers for disease diagnosis, prognosis, and treatment monitoring. ${ }^{21}$

Recently, it was found that circulating miRNAs were dysregulated in the mdx mouse, in the CXMDj (canine $\mathrm{X}$-linked muscular dystrophy in Japan) dog (beagle dogs harboring the GRMD mutation), and in human DMD and Becker muscular dystrophyBMD patients (K. Wahbi, F. Amor, L. Jeanson-Leh, N. Vignier, A. Béhin, T. Stojkovic, G. Bonne, T. Voit, D. Israeli, unpublished data). ${ }^{22-26}$ These circulating miRNAs included miR-1, miR-133a, miR-133b, miR-206, and miR-378 (muscle miRNA, designated dystromiRs), which are all up-regulated in the serum of affected animals and patients with dystrophin deficiency, probably because of their leakage into the circulation after muscle fiber damage, similar to CK. Oversecretion of exosomes and microparticles from dystrophin-deficient tissues are another potential source of miRNA release into the circulation. ${ }^{27,28}$

Studies on various cardiac pathologies previously identified circulating miRNA biomarkers for cardiac dysfunction, ${ }^{29}$ including miR-208b and miR-499, which are expressed in both heart and skeletal muscles, and the heartspecific miR-208a. ${ }^{30-32}$ However, an evaluation of serum miRNA biomarkers for cardiac pathology in dystrophic animals and patients has not yet been reported.

In the first phase of this study we used a miRNA highthroughput sequencing (HTS) technology for a comprehensive identification of dysregulated miRNAs in the serum in GRMD dogs. In a second study phase, a subselection of the identified dysregulated miRNAs was characterized in details in a large-scale (using hundreds of serum samples) longitudinal study. Finally, the dysregulation of cardiacenriched miRNAs was evaluated in a functional investigation in GRMD dogs, and their dysregulation was confirmed in a small cohort of DMD patients.

\section{Materials and Methods}

\section{Ethical Declaration}

All dog and mouse procedures were performed in accordance with local ethics committees [Ethical Committee of the Région des Pays de la Loire, University of Angers, France, the common Ethical Committee of ANSES (Agence nationale de sécurité sanitaire de l'alimentation, de l'environnement et du travail)/ UPEC (Université Paris-Est Créteil)/ENVA (Ecole Nationale Vétérinaire d'Alfort), and Ethical Committee of Généthon, Evry, France]. Animals were handled according to A1 biosafety requirements and in accordance with the European guidelines for use of experimental animals (L358-86/609/EEC). All experiments were performed to minimize animal discomfort.

The human study (DMD patients and controls) was conducted according the principles of the Declaration of Helsinki ethical principles for medical research and was specifically approved by the Ethical Committee CPP Ile de France VI, July 20, 2010, and the Comité d'Ethique (412) du CHR La Citadelle (Liège, Belgium) January 26, 2011. 


\section{Biological Samples}

Mice

The mouse strains included were the dystrophin-null mdx CV4 and its genetic background control C57BL/6J. Before blood extraction, mice were anesthetized by intraperitoneal injection of ketamine/xylazine. Anesthetized mice were sacrificed by cervical elongation at the end of the experiments. Blood samples were collected into nonheparinized tubes in the absence of anticoagulation treatment. Fresh blood samples were allowed to coagulate 30 minutes at room temperature and spun down at $1800 \times g$ for 10 minutes. Supernatant fluid was collected into fresh tubes and stored at $-80^{\circ} \mathrm{C}$ until further processing.

Dogs

Sera of dogs $<60$ days old were obtained from the Domaine des Souches breeding center (Mézilles, France). Sera of dogs $>60$ days old were obtained from the GRMD colonies maintained in the Boisbonne Center for Gene Therapy of the National Veterinary School of Nantes (Atlantic Gene Therapy, ONIRIS, Nantes, France) or from the National Veterinary School of Alfort (Maisons-Alfort, France).

Dog cardiac and skeletal muscle (ulnar lateral muscle) tissues were taken from 6- to 7-month-old GRMD dogs and controls.

Cardiac TpnI was measured in dog serum samples by using the dedicated kit for the Immulite 2000 analyzer (reference L2KTI; Siemens Healthcare, Malvern, PA). The analytical sensitivity of this test was $0.2 \mathrm{ng} / \mathrm{mL}$, and the maximum measurable concentration was $180 \mathrm{ng} / \mathrm{mL}$. For some dogs with elevated values, a dilution of the serum samples was made by using serum from a healthy dog with confirmed undetectable TpnI.

For the cardiac function study, 17 GRMD dogs aged 9.6 to 13.7 months old (mean age, $11.6 \pm 1.5$ months) underwent a blood sample (miRNA and TpnI levels) assessment synchronized with an echocardiographic examination combined with two-dimensional color Doppler tissue imaging, as previously described. ${ }^{33}$ Briefly, the following parameters were calculated: end-systolic and end-diastolic left ventricular diameters, left ventricular free wall and septum thicknesses; left ventricular fractional shortening (FS) was calculated as

\footnotetext{
[left ventricular end-diastolic diameter - left ventricular end-systolic diameter]/left ventricular end-diastolic diameter $\times 100 \%$.
}

Pulsed-wave Doppler velocities were also measured, notably the mitral E wave-to-A wave ratio was calculated. Doppler tissue imaging examination provided radial left ventricular free wall motion velocities and endoepicardial systolic gradients. The correlation between miRNA levels, TpnI, and all the obtained echocardiographic parameters were first assessed. In a second time the 17 GRMD dogs were divided in two groups according to their FS value, as a quantitative measure of their left ventricular contractile dysfunction. The threshold was set at $30 \%$ (normal values obtained in healthy littermates range from $37 \%$ to $42.5 \%{ }^{34}$ ), 12 of 17 GRMD dogs had a FS $<30 \%$ (ranging from $20.7 \%$ to $29.8 \%$; mean age, $11.4 \pm 1.2$ months), 5 of 17 had a FS $>30 \%$ (ranging from $30.6 \%$ to $36.4 \%$; mean age, $11.3 \pm 1.5$ months). A group effect on the level of TpnI and candidate cardiac miRNAs was assessed.

\section{Human Patients and Controls}

Blood samples were obtained after informed consent at CPP Ile de France VI on July 20, 2010, and at the Comité d'Ethique (412) du CHR La Citadelle (Liège, Belgium) on January 26, 2011. Patient inclusion criterion was genetically proven DMD diagnosis. According to the ethical requirements, human blood samples were collected from male subjects $>3$ years old weighing at least $15 \mathrm{~kg}$ for control and for genetically confirmed DMD patients. Peripheral blood samples were collected into 5-mL K3EDTA tubes (Greiner Bio-One, Longwood, FL). Plasma was separated from buffy coat and red blood cells after 10 minutes of centrifugation at $1800 \times g$ and stored at $-80^{\circ} \mathrm{C}$ until further processing.

Human skeletal muscle tissues were obtained from the Myobank, the tissue bank of the Association Francaise contre les Myopathies. Open skeletal muscle biopsies were performed, after informed consent, according to the Declaration of Helsinki. Muscle biopsies included in this study were derived from the paravertebral muscle (three controls and two DMD donors) and the dorsal muscles (one DMD donor).

\section{RNA Extraction and Quality Control Procedures}

In preliminary experiments (data not shown), the miRNeasy (Qiagen, Valencia, CA) and miRVana PARIS (Ambion, Austin, TX) RNA extraction kits were identified as suitable for our experimental system for dog serum and human plasma, respectively. Total RNA extraction was performed from 300 $\mu \mathrm{L}$ of dog serum or $600 \mu \mathrm{L}$ of human plasma. RNA was eluted in $100 \mu \mathrm{L}$ of RNase-free water, precipitated overnight, and resuspended in $10 \mu \mathrm{L}$ of RNase-free water. Total RNA was quantified by using a Nanodrop spectrophotometer (ND8000; Labtech, Wilmington, DE) and analyzed with the Agilent small and pico RNA kit in the 2100 Bioanalyzer (Agilent Technologies, Santa Clara, CA). Other RNA samples used in this study are dog total RNA from skeletal muscle, heart, and brain (Zyagen, San Diego, CA) and human total RNA from skeletal muscle, heart, and brain (Ambion).

\section{miRNA Sequencing}

miRNA sequencing was performed by Integragen (Evry, France). Libraries were constructed as described, ${ }^{35}$ with some modifications for efficiency improvement in small samples. Briefly, a $3^{\prime}$ adenylated DNA adaptor was ligated in the presence of $12 \%$ polyethylene glycol and the absence of ATP, avoiding miRNAs self-ligation. A 5' RNA adaptor was ligated in the presence of ATP. Reverse transcription primer complementary to the $3^{\prime}$ adaptor was added, forming a duplex to 
reduce adapter dimer formation. Reverse transcription reaction was done with 1.75 pmol adaptors ( $3^{\prime}$ adaptor $/ 5^{\prime}$ adaptor/ reverse transcription primer), and cDNAs were amplified by 13 PCR cycles with primers complementary to the $3^{\prime}$ and $5^{\prime}$ adaptors. During this PCR step, a specific barcode was incorporated for individual sample recognition. PCR sample band quantification was done with Fragment Analyzer (Advanced Analytical Technologies, Inc., Ames, IA). An equimolar pool of 10 different samples migrated on PAGE, and the miRNA band was extracted (MinElute column; Qiagen). Libraries were quantified by real-time quantitative PCR (qPCR), to load precisely $7 \mathrm{pmol} / \mathrm{L}$ pool per line of HiSeq Flow-Cell. The HiSeq 36b and index (barcode) sequencing was done as instructed (Illumina, San Diego, CA) with a SBS V3 kit leading on 150 million passing filter clones. Unique miRNA reads and their copy numbers were analyzed with miRanalyzer online software (http://bioinfo2.ugr.es/miRana lyzer/miRanalyzer.php), ${ }^{36}$ using dog, human, and mouse miRbases as references (19th miRbase, accessed August 2012). Only miRNA with $>10$ reads in at least half of the animals were considered as expressed.

\section{RT-qPCR}

miRNAs were quantified by quantitative RT-PCR (RTqPCR) with technologies from both Applied Biosystems (AB; Foster City, CA) and Exiqon (Woburn, MA).

\section{AB Technology/Multiplex}

Total RNA (350 ng) was reverse-transcribed with the Megaplex Primer Pools A and B (human version 3), and miRNAs were quantified with TaqMan Array MicroRNA Cards A and B (human version 3) on the 7900HT RealTime PCR System (AB) according to the manufacturer's guidelines. Quantification cycle $(\mathrm{Cq})$ values were calculated with the SDS software version $2.3(\mathrm{AB})$ by using automatic baseline with a threshold fixed at 0.1 .

\section{AB Technology/Simplex}

Total RNA (10 ng or $50 \mathrm{ng}$ in the case of miR-208a) was converted to cDNA via miRNA-specific stem loop reverse transcription primers (Applied Biosystems miRNA assays). cDNA was diluted $8 \times$ or $4 \times$ in the case of miR-208a and quantified with miRNA-specific primers and Taqman probes by using the 7900HT Real-Time PCR system (AB). Cq values were calculated with the SDS software version $2.3(\mathrm{AB})$ by using automatic baseline with a threshold fixed at 0.2 .

\section{Exiqon Technology/Simplex}

Total RNA (20 ng) was converted into poly-A-primed universal cDNA, and miRNAs were quantified with miRNAspecific locked nucleic acid primers on the 7900HT RealTime PCR System (AB) according to the manufacturer's guidelines. Cq values were calculated with the SDS software version $2.3(\mathrm{AB})$ by using automatic baseline with a threshold fixed at 0.2 .

\section{Data Processing and Statistical Analysis}

miRNA abundances were calculated from the HTS data by normalizing unique miRNA read counts relative to the total read count per serum sample. miRNA expression RT-qPCR results, expressed as raw $\mathrm{Cq}$ with a threshold $\mathrm{Cq} \leq 35$, were normalized in the large-scale screenings to the calculated mean $\mathrm{Cq}$ of the sample (21) and to miR-16 for individual assays. Differential expression was calculated with the 2- $\Delta \mathrm{Ct}$ method, and miRNAs were considered differentially expressed beyond a threshold of a two-fold change.

\section{Results}

DystromiRs, miR-95, Cardiac Muscle miRNAs, and the DLK1-DIO3 miRNA Cluster Are Dysregulated in the Serum of GRMD Dogs

The HTS technology was used to profile and compare miRNA expression in the serum of 6-week-old GRMD dogs with control dogs $(n=5)$. Mean read count was approximately 1.2 million miRNAs per GRMD and 0.8 million miRNAs per control dog sample. Recovered sequences were analyzed by miRAnalyzer. ${ }^{36}$ We took advantage of the strong homology between human and dog and between mouse and dog complete miRNA content (perfect identity of 57\% and 47\%, respectively; 19th miRbase, August 2012) to match the obtained sequences with the dog, human, and mouse miRbases. Among a total of 430 expressed miRNA species, 198 (46\%) were referenced in the dog miRbase and $232(54 \%)$ were not, but they were identified by identity to their human and mouse orthologs. A list of all detected miRNAs in dog serum is presented in Supplemental Table S1. Dysregulated miRNAs in GRMD dogs are presented graphically in Figure 1 and Supplemental Tables S2 and S3 for the up- and down-regulated miRNAs in GRMD dog serum, respectively.

Among the up-regulated miRNAs we found the previously identified dystromiRs miR-1, miR-133a, miR-133b, miR-206, and miR-378, ${ }^{22-24}$ serving as an internal positive control for this approach. Similarly, we identified a dysregulation of another skeletal muscle-enriched miRNA, miR-95, which is located in an intron of the Ablim-2 gene that encodes a muscle actin-interacting protein. ${ }^{37}$

A second identified class of dysregulated miRNAs in the serum of GRMD dogs included a large number of members of the DLK1-DIO3 locus. This locus, which contains the largest miRNA cluster in mammalian species ${ }^{38}$ is involved in the muscular hypertrophy Callipyge phenotype in sheep ${ }^{39,40}$ and was recently found to be transcriptionally up-regulated by MEF2A in regenerating skeletal muscle in mice. ${ }^{41}$ Sixteen DLK1-DIO3 miRNAs (20, considering isomiRNAs and both pre-miRNA strands) were highly dysregulated ( $>10$-fold), including miR-136 (5p, and two 3p-isomiRNAs), miR-369, miR-377, miR-381, miR-410, miR-412, miR-431 (both 5p and 3p), miR-433, miR-487b, miR-495, miR-543, miR-655, 


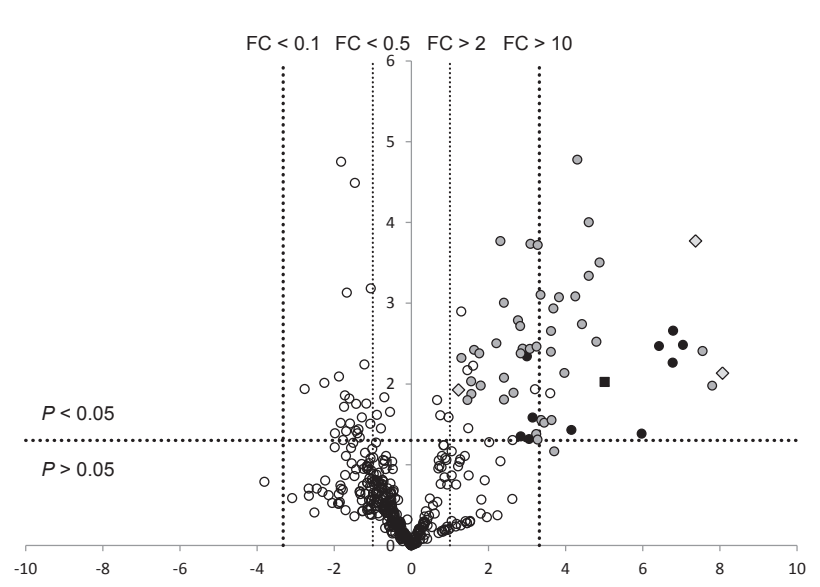

Figure 1 Large-scale miRNA sequence screening for dysregulated serum miRNAs in golden retriever muscular dystrophy (GRMD) dogs. Dogs were compared in miRNA sequence analysis [high-throughput sequencing (HTS) focused on miRNA]. Dogs were all 1.5 months old. Dots correspond to dystromiRs (black dots), DLK1-DI03 clustered miRNAs (gray dots), miR-95 (black squares), and cardiac miRNAs (gray diamonds). miRNAs upregulated in the serum in GRMD dogs $>10$-fold $(\mathrm{FC}>10 ; P<0.05)$ are plotted in the upper right box. No strongly ( $>10$ fold) down-regulated miRNAs could be identified. FCs of relative miRNA abundances and related $P$ values are represented in a volcano plot. Vertical and horizontal dot lines indicate $\mathrm{FC}=10$ (negative and positive) and $P=0.05$ (Student's $t$-test), respectively. $n=5$ GRMD dogs; $n=5$ control dogs.

miR-656, miR-889, miR-411 (two 5p-isomiRNAs), and miR539. Other 13 DLK1-DIO3 miRNAs (21, considering isomiRNAs) were up-regulated to a smaller level (2- to 10-fold; $P<0.05)$, and an additional 4 miRNAs were not significantly $(P>0.05)$ up-regulated. From a total of 52 known $D L K 1$ DIO3-clustered miRNAs (according to human miRbase), the expression of only 3 was unchanged in the serum in GRMD dogs compared with control dogs and 16 were not detected in dog serum.

A third identified class of dysregulated miRNAs in the serum of GRMD dogs was heart-enriched miRNAs, including miR-208a, miR-208b, and miR-499 (Figure 1 and Supplemental Table S2).

\section{Longitudinal Investigation of Dysregulated DystromiRs and DLK1-DIO3 miRNAs in the Serum of GRMD Dogs}

A subselection of the dysregulated miRNAs was further subjected to an individual RT-qPCR investigation. We used a large collection of archived serum samples of GRMD and control dogs aged between 5 days and 1 year in a large-scale longitudinal expression study. The number of studied dogs and samples is detailed in Supplemental Table S4. For analytical purposes these samples were divided into four age groups: $<1$ month, 1.5 to 3.5 months, 4 to 7 months, and 7.2 to 12 months. Relatively stable expression of miR-16 was confirmed in GRMD and healthy control dogs (Supplemental Figure S1) and was thus used as an internal normalization control in all subsequent experiments. We observed an upregulation of all tested dystromiRs, significantly in the youngest dog group ( $<1$ month old) only for miR-1, but increasingly and significantly up-regulated for all four dystromiRs in all older GRMD age groups (Figure 2 and Table 1). Receiver operating characteristic (ROC) curve analyses in the respective age groups found high discriminative values for all dystromiRs (Figure 2 and Table 2), most of them being comparable with CK (Figure 2).

miR-95 was up-regulated in the serum of most tested GRMD animals, except in the younger (non-dysregulated) and the older (up-regulated $2 \times$, nonsignificant) age groups (Figure 2 and Table 1). The DLKI-DIO3 clustered miRNAs, miR-410 and miR-431, were significantly up-regulated already in the younger GRMD dog group ( $<1$ month) and increasingly up-regulated in older GRMD dogs (Figure 2 and Table 1). Interestingly, expression of miR-410 was downregulated with age in control dog samples immediately after birth, whereas in the GRMD dogs the highest expression level was detected in the 1.5- to 3.5-month age group, in correlation with the disease onset, and was subsequently down-regulated with age at later time points. Up-regulation in GRMD dogs and down-regulation with age in both GRMD and control dogs were also observed with miR-431, although to a lesser extent. Expression patterns of five DLK1-DIO3 locus miRNAs correlate strongly (Supplemental Table S5), but neither with the normalizer miR-16 nor with the upregulated miR-95, suggesting their co-regulation within the DLK1-DIO3 cluster.

Longitudinal Investigation of Cardiac-Enriched miRNA Dysregulation in the Serum of GRMD Dogs

miR-208a, miR-208b, and miR-499 were recently found to be dysregulated in the serum of patients in a variety of ischemic cardiovascular disorders. ${ }^{30,32,42}$ In a previous study on the $m d x$ mouse dystrophic model, these miRNAs were not dysregulated, ${ }^{22}$ which was expected, given that young mdx mice present only mild, if any, cardiomyopathy. Similar to DMD patients, GRMD dogs present a significant cardiomyopathy, ${ }^{3}$ thereby providing an opportunity to identify dysregulated miRNAs associated to this pathology.

The initial HTS screening identified a strong and significant up-regulation of the cardiac-enriched miRNAs miR-208a and miR-208b in GRMD dog serum (Figure 1) and a milder upregulation of miR-499 $(2 \times ; P<0.02)$ (Supplemental Table $\mathrm{S} 2)$. We used the longitudinal cohort serum samples to analyze the expression of these cardiac-enriched miRNAs by RT-qPCR. miR-499 was expressed in the serum of all studied animals and was up-regulated significantly in dogs older than 1.5 months, with the higher fold change (FC) values in the 4- to 7-month age group (Figure 3 and Table 1). miR-208b, which was hardly detectable in healthy dogs, was clearly detected in most GRMD dogs of all age groups and was up-regulated significantly in dogs older than 1.5 months with highly discriminative FC and ROC curves values. miR-208a expression, which was rarely detected in healthy dogs, was detected in almost all GRMD dogs older than 1.5 months and was significantly up-regulated. 
A

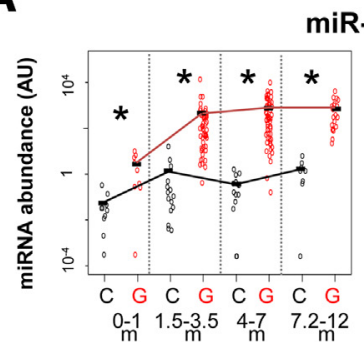

miR-1

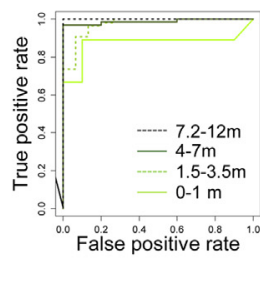

C

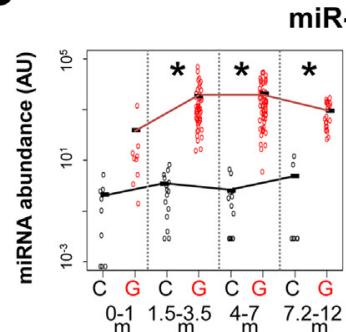

miR-206

E

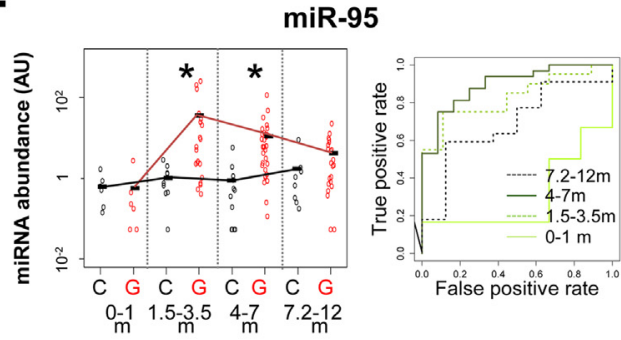

G

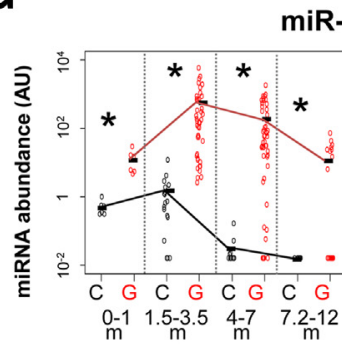

$\operatorname{miR}-431$

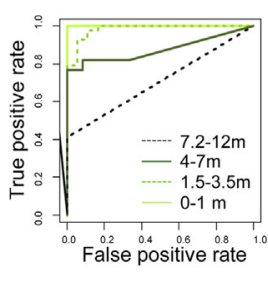

I
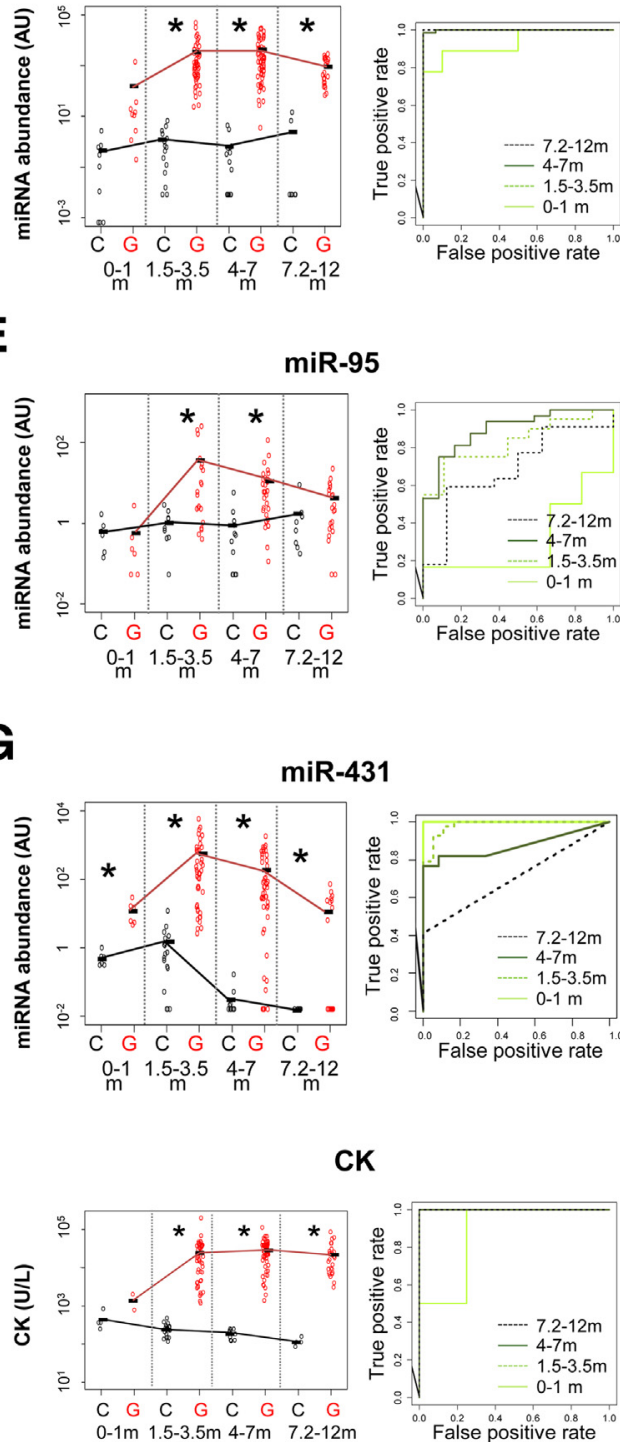

$F$

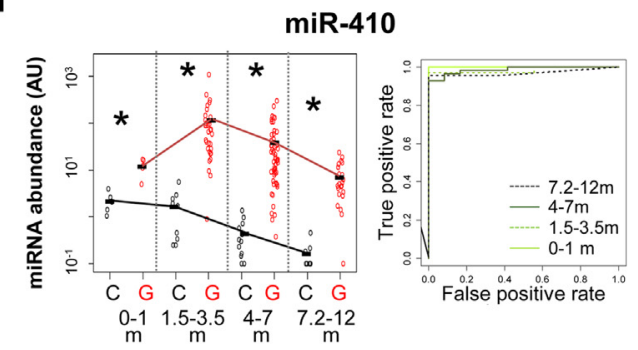

H
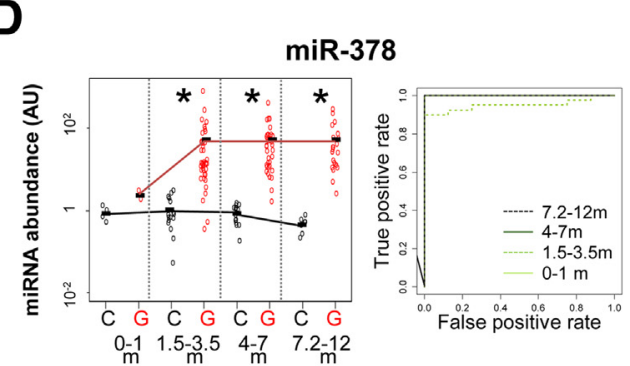

F
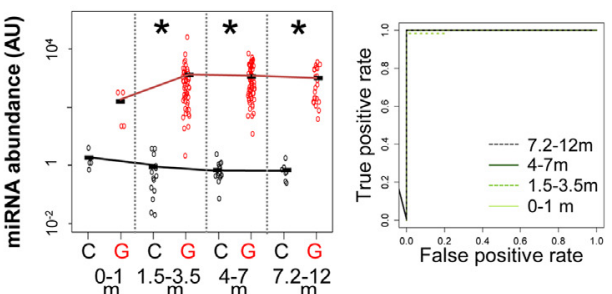

B $\begin{array}{cccc}0-1 & 1.5-3.5 & 4-7 & 7.2-12 \\ \mathrm{~m} & \mathrm{~m} & & \end{array}$

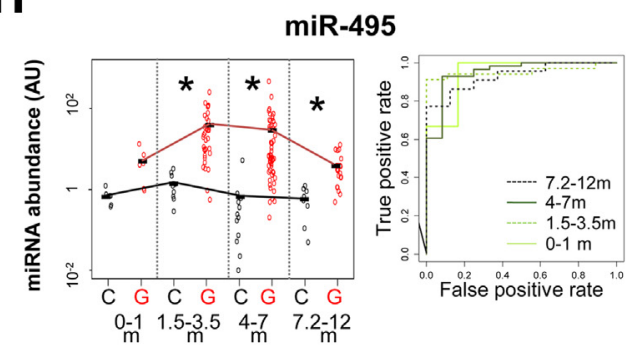

Figure 2 Age-dependent expression of dystromiRs and DLK1-DI03 miRNAs in serum in GRMD dogs. DystromiRs (A-D), miR-95 (E), DLK1-DI03 miRNAs $(\mathbf{F}-\mathbf{H})$, and CK (I) were quantified in a collection of sera of golden retriever muscular dystrophy (GRMD; red dots) and control (black dots) dogs in four age groups of 0 to1, 1.5 to 3.5, 4 to 7, and 7.2 to 12 months. F-G: For DLK1-DIO3 miRNA levels were normalized to miR-16 and expressed in abundance AUs. Each dot represents one dog. Mean value per group is given as a black bar. ROC curves are shown for the same four age groups (birth to 1, 1.5 to 3.5, 4 to 7, and 7.2 to 12 months), and the corresponding area under the curve values are indicated. Fold change, $P$ values, and ROC curve values per age group are shown in Tables 1 and 2, respectively. Median, mean, and SD of all tested miRNAs and dog age groups are given in Supplemental Table S7. $n=78$ to 81 GRMD dogs (miRNA dependent) and 102 to 150 of their serum samples (A-D); $n=27$ to 31 control dogs (miRNA dependent) and 43 to 48 of their serum samples (A-D); $n=34$ GRMD dogs and 80 serum samples (E); $n=19$ control dogs and 35 control serum samples (E); $n=49$ to 58 GRMD dogs (miRNA dependent) and 118 to 127 of their serum samples $(\mathbf{F}-\mathbf{H}) ; n=84$ GRMD dogs and 153 of their serum samples $(\mathbf{I}) ; n=22$ control dogs and 38 of their serum samples (I). ${ }^{\star} P<0.05$, Student's $t$-test, between control and GRMD groups. AU, arbitrary unit; C, control; G, GRMD. 
Table 1 FC and Significance (Per Age Group) of Serum Dysregulated miRNAs in GRMD Dogs

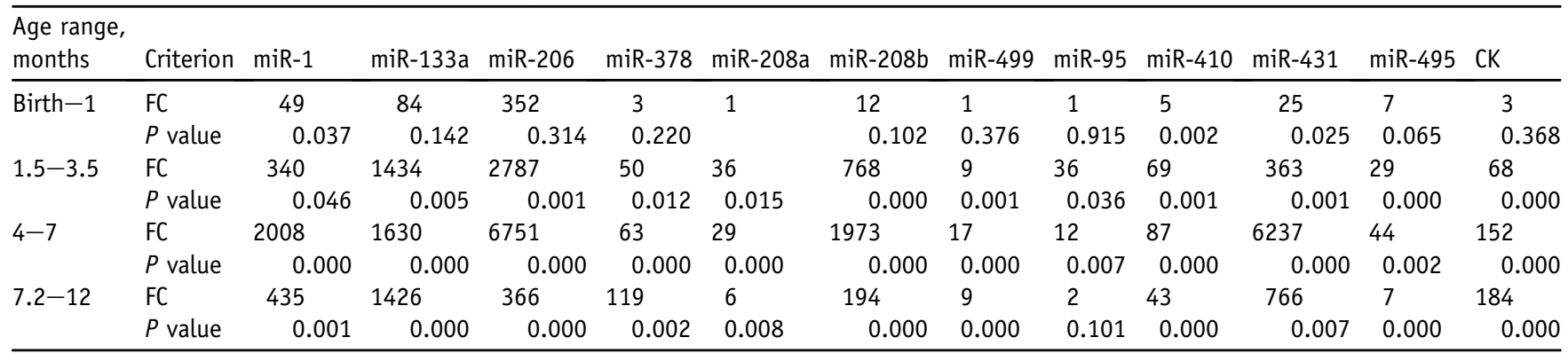

Values in this table correspond to data presented in Figures 2 and 3.

CK, creatine kinase; FC, fold change; GRMD, golden retriever muscular dystrophy.

\section{Cardiac-Enriched miRNAs and Cardiac Tpn Levels Do Not Correlate in GRMD Dogs with Cardiac Functional Assessment}

We next aimed at evaluating the correlation of cardiacenriched miRNAs to cardiomyopathy in GRMD dog serum. Cardiac Tpn and miRNAs were quantified in the serum of two groups of young adult (mean age, 11.6 months; range, 9.6 to 13.7 months) GRMD dogs, the first with cardiac FS $\geq 30 \%(n=5)$, the second with cardiac FS $<30 \%$ $(n=12)$. A third group of healthy dogs was used as control $(n=8)$. Significant up-regulation of the studied miRNAs in GRMD dogs (the two groups combined) was confirmed (Figure 4A), which was in agreement with the longitudinal study (Figure 3). However, none of these markers were expressed differentially between the two GRMD dog groups (with different cardiac FSs). Similarly, correlation could not be identified between expression levels of these markers (serum miRNAs and cardiac Tpn) to several other cardiac functional tests, including end-systolic and end-diastolic left ventricular diameters, left ventricular free wall and septum thicknesses, pulsed-wave Doppler velocities, and Doppler tissue imaging examination (data not shown). Despite a high up-regulation average level of cardiac Tpn in GRMD dogs compared with control dogs $(>200 \times)$, it was not significant in our cohort (Figure 4A), explained because some GRMD dogs (both with high and low values of FS) were cardiac Tpn negative. In the same cohort, some GRMD dogs were almost or completely negative also for miR-208a, which, however, was significantly different between the control and GRMD dog groups. This raised the hypothesis that the level of these cardiac biomarkers may fluctuate over time in this chronic cardiomyopathy. We thus analyzed miRNA expression levels over time in individual GRMD dogs, at the ages of 2, 4, 6, 9, and 12 months, and confirmed this fluctuating pattern for miR-208a (Figure 4B), providing evidence that GRMD dogs with cardiomyopathy may shift in time between periods of miR-208a positive and negative, obviously not in correlation to left ventricular functional parameters. In contrast, the expression of miR-208b and miR-499 in these individual GRMD dogs remained stably up-regulated at all studied time points. Finally, we analyzed in this cohort the correlation between cardiac miRNA expression levels and CK or cardiac Tpn, which are the reference serum protein biomarkers for diseased skeletal and cardiac muscles, respectively (Figure 4C). We identified positive correlations of the three cardiac-enriched miRNAs to CK expression, whereas the expression of cardiac Tpn correlated mildly only with miR-208a.

\section{Serum Expression of the DLK1-DIO3 Locus miRNAs in the $m d x$ Mouse and in DMD Patients}

A selection of the DLK1-DIO3 locus miRNAs (miR-410, miR433, miR-494, miR-495, and miR-539) was studied further in the $m d x$ mouse and in DMD patients (Supplemental Table S6). The five DLKI-DIO3 miRNAs were significantly up-regulated in 1-month-old and to a lesser extent in 5-month-old $m d x$ mice (Figure 5, A and B). Their expression was however significantly down-regulated with aging in both control C57Bl/6 and $m d x$ mice (Figure 5, A and B), which is in agreement with the data obtained in GRMD dogs. The same miRNAs, as well as miR-95

Table 2 AUC Values (Per Age Group) of Serum Dysregulated miRNAs in GRMD Dogs

\begin{tabular}{|c|c|c|c|c|c|c|c|c|c|c|c|c|}
\hline $\begin{array}{l}\text { Age range, } \\
\text { months }\end{array}$ & $\operatorname{miR}-1$ & miR-133a & miR-206 & miR-378 & miR-208a & miR-208b & miR-499 & miR-95 & $\operatorname{miR}-410$ & miR-431 & miR-495 & CK \\
\hline Birth-1 & 0.872 & 1 & 0.933 & 1 & 0.5 & 0.889 & 0.694 & 0.306 & 1 & 1 & 0.944 & 0.875 \\
\hline $1.5-3.5$ & 0.972 & 0.996 & 1 & 0.95 & 0.733 & 0.986 & 0.9 & 0.828 & 0.984 & 0.983 & 0.954 & 1 \\
\hline $4-7$ & 0.987 & 1 & 0.999 & 1 & 0.746 & 0.989 & 0.969 & 0.896 & 0.987 & 0.876 & 0.949 & 1 \\
\hline $7.2-12$ & 1 & 1 & 1 & 1 & 0.656 & 0.989 & 0.977 & 0.687 & 0.969 & 0.705 & 0.932 & 1 \\
\hline
\end{tabular}

Values in this table correspond to data presented in Figures 2 and 3.

AUC, area under the curve; CK, creatine kinase; GRMD, golden retriever muscular dystrophy. 
A
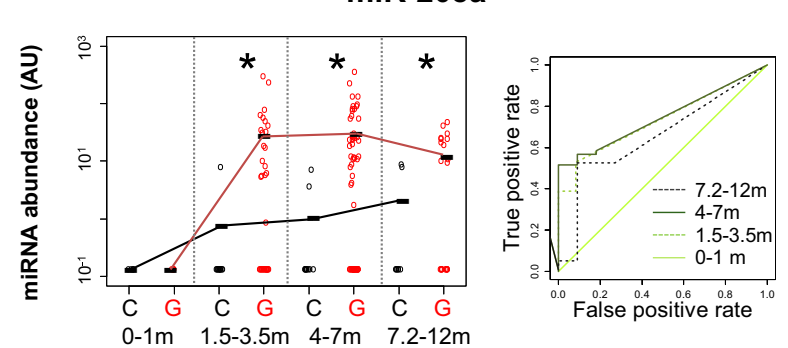

B
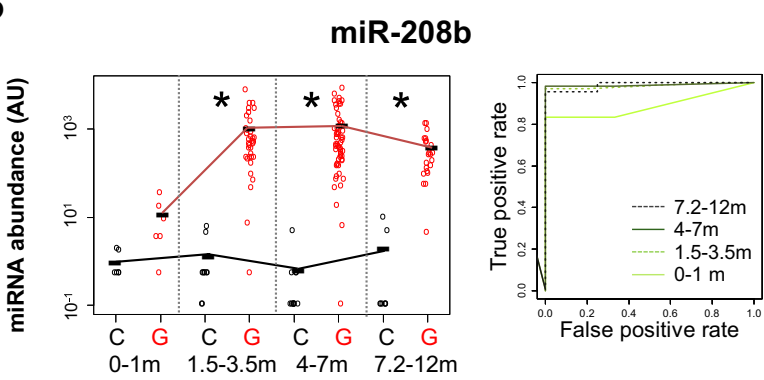

C
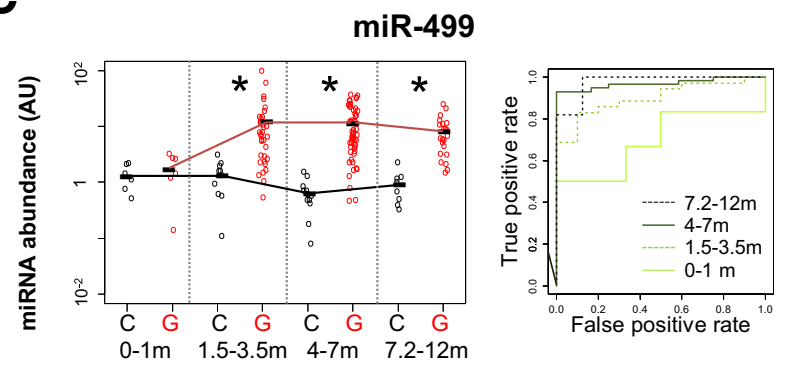

Figure 3 Age-dependent expression of cardiac-enriched miRNAs in the serum in golden retriever muscular dystrophy (GRMD) dogs. miR-208a (A), miR-208b (B), and miR-499 (C) were quantified in a collection of sera derived from GRMD (red dots) and control (black dots) dogs from age groups of birth to $1,1.5$ to $3.5,4$ to 7 , and 7.2 to 12 months. Fold change and $P$ values (Student's $t$-test) and receiver operating characteristic curve values per age group are shown in Tables 1 and 2, respectively. Median, mean, and SD of all tested miRNAs and dog age-groups are given in Supplemental Table S7. $n=51$ individual dogs and 120 plasma samples (A-C, GRMD cohort); $n=19$ individual healthy dogs and 35 serum samples (A-C, control cohort). ${ }^{*} P \leq 0.05$ difference in expression levels of GRMD versus control dogs. AU, arbitrary unit; $C$, control; G, GRMD.

(which exists in humans and dogs but not in mice) were tested in plasma samples derived from 5- to 7-year-old DMD patients $(n=5)$ and age-matched $(n=3)$ controls (Supplemental Table S7). Up-regulation of miR-95 was validated in DMD patients and was similar to the pattern observed in GRMD dogs. In contrast, slight down-regulation (miR-539) or similar levels (miR-410, miR-433, miR-494) of the DLK1-DIO3 locus miRNAs were found in the DMD patients (Figure 5, C and D).

Serum Expression of Cardiac-Enriched miRNAs in the $m d x$ Mouse and in DMD Patients

In addition, we analyzed miR-208a, miR-208b, and miR499 in $m d x$ mouse and in DMD patients compared with their respective controls. In $m d x$ mice, heart-enriched miRNAs in the serum were not significantly dysregulated (Figure 6A). In humans miR-499 was expressed in both controls and DMD patients, whereas miR-208a and miR-208b were detected exclusively in DMD patients, in three of five for miR-208a and in all five DMD patients for miR-208b (Figure 6B). Up-regulation was significant for miR-499 and miR-208b but not for miR-208a.

\section{Cardiac-Enriched miRNA Expression in Skeletal and Cardiac Muscle Tissues}

We finally evaluated the expression of the cardiac-enriched miRNAs in skeletal muscles in DMD patients versus controls, and in both skeletal and cardiac muscles in GRMD dogs compared with controls. We confirmed the expression of miR-208b and miR-499 in skeletal muscles of both DMD patient and controls, miR-499 being down-regulated in DMD muscle (FC $=-6.3 ; P \leq 0.03$ ). Unexpectedly, we also detected the expression of miR-208a in the same biopsies (Figure 7), in a level only slightly lower than miR208b (miR-208b/miR-208a $=6.3$ and 3.2; $P<0.05$ in controls and DMD patients, respectively). Expression of these three miRNAs was also detected in dog skeletal muscles (Figure 7). However, expression of miR-208a was largely lower than expression of miR-208b ( $>35$-fold) both in cardiac and skeletal muscles, both in GRMD and control dogs. Most importantly, in GRMD dogs, miR-208a expression was only 1.7-fold higher in heart muscles than in skeletal muscles.

\section{Discussion}

Profiling circulating miRNAs has emerged in recent years as a promising technology for disease diagnosis and monitoring. There are only a few reports in the literature on circulating miRNAs in muscle diseases. A dysregulation of the dystromiRs in DMD, including miR-1, miR-133, and miR-206, described initially by Bozzoni et al, ${ }^{23}$ was later confirmed in the dystrophic beagle dog model. ${ }^{24}$ These two studies used a miRNA discovery-targeted approach rather than a systematic screening of complete miRNA content. More recently, we have performed a large-scale screening of complete miRNA content in a collection of mouse models, including the DMDmodel $m d x$ mouse, resulting in the identification of some additional dysregulated miRNAs in DMD, including the miRNAs miR-378, miR-378*, and miR-31. ${ }^{22}$ However, the $m d x$ mouse is characterized by a mild dystrophic phenotype in skeletal muscles and a mild cardiac phenotype, raising the possibility that some DMD dysregulated miRNAs remained undetected in this model.

We therefore focused on the identification of dysregulated circulating miRNAs in a clinically more relevant DMD model, the GRMD dog. A screening was performed with miRNA HTS technology, and the obtained 

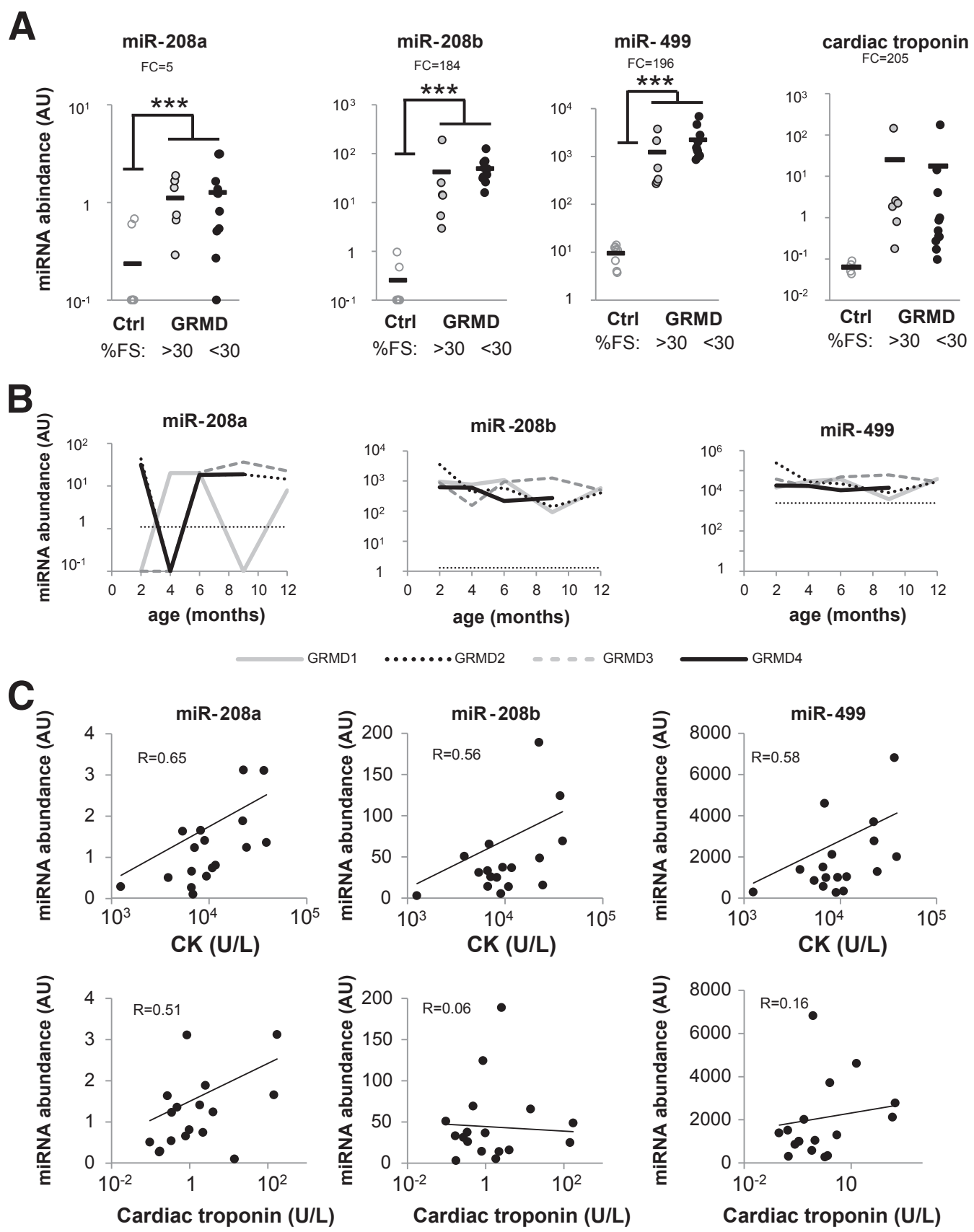

Figure 4 Serum expression of cardiac-Tpn and cardiac-enriched miRNAs does not correlate in golden retriever muscular dystrophy (GRMD) dogs with left ventricular functional assessment. Cardiac FS (a left ventricular functional test), cardiac Tpn, and serum miRNAs evaluation in 1-year-old GRMD dogs. A: miRNAs abundance and cardiac-Tpn in GRMD dogs with low FS $(<30 \%)$, high FS $(\geq 30 \%)$, and control healthy dogs. FCs are indicated on the graphs. B: miRNA level in GRMD dogs of the ages 2, 4, 6, 8, and 12 months. C: Correlation of serum cardiac-enriched miRNAs with CK and cardiac Tpn in 1-year-old GRMD dogs. Pearson's correlation coefficient $R$ values are indicated on the graphs. Corresponding $P$ values are as follows: miR-208a/CK, $P=0.004 ; \mathrm{miR}-208 \mathrm{~b} / \mathrm{CK}$, $P=0.021 ; \mathrm{miR}-499 / \mathrm{CK}, P=0.015 ; \mathrm{miR}-208 \mathrm{a} / \mathrm{CT}, P=0.037$; miR-208b/CT, $P=0.823$; and miR-499/CT, $P=0.54$. Median, mean, and SD of all tested miRNAs for the dog groups are given in Supplemental Table S8. $n=13$, GRMD dogs with low FS $(\mathbf{A}) ; n=6$, GRMD dogs with high FS (A); $n=8$, control healthy dogs (A); $n=17$, GRMD dogs (C). ${ }^{* *} P<0.001$ (miR-208a, $P=0.0005 ;$ miR-208b, $P=0.0009$; miR-499, $P=0.0005 ;$ and $\left.\mathrm{CT}, P=0.13\right)(\mathbf{A})$. AU, arbitrary unit; Ctrl, control; $\mathrm{CT}$, cardiac troponin.

sequences were matched against dog, human, and mouse databases to cover as efficiently as possible the yet uncompleted dog miRNA content. In comparison to the observed dysregulation in dystrophic subjects in previous studies, ${ }^{22-24}$ high FC values were obtained for the dystromiRs in the GRMD dog model. With FC ranging from
50 to 1000 between GRMD and controls dogs, the GRMD model displays one of the highest levels of serum miRNA dysregulation in dystrophic animal models and patients, reported so far.

The dystromiRs are myofiber-enriched miRNA species, which are dysregulated in muscular dystrophies. ${ }^{22-25}$ To the 
A

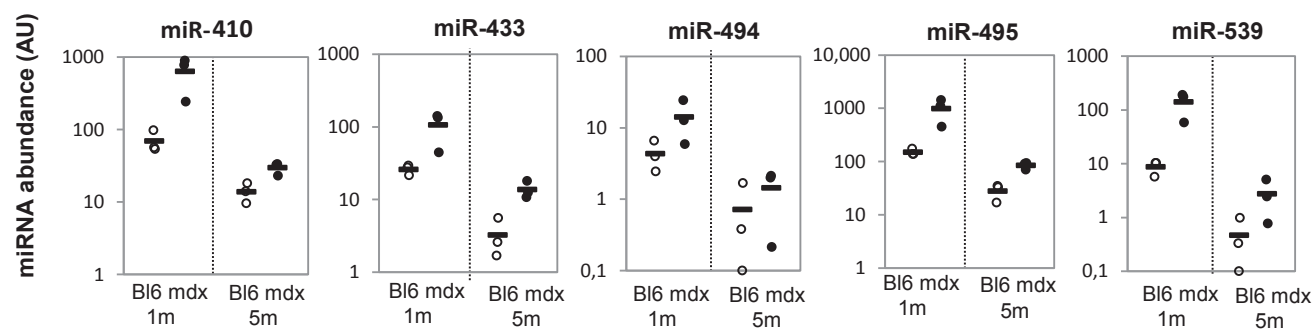

B

\begin{tabular}{|c|c|c|c|c|c|}
\cline { 3 - 6 } \multicolumn{2}{c|}{} & \multicolumn{2}{c|}{ Upregulated in mdx } & \multicolumn{2}{c|}{ Downregulated with age } \\
\cline { 3 - 6 } \multicolumn{2}{c|}{} & 1 month & 5 months & C57B//6 & $\mathrm{mdx}$ \\
\hline \multirow{2}{*}{ miR-410 } & $\mathrm{FC}$ & 9 & 2 & -5 & -21 \\
\cline { 2 - 6 } & pval & 0.05 & 0.09 & 0.05 & 0.09 \\
\hline \multirow{2}{*}{ miR-433 } & $\mathrm{FC}$ & 4 & 4 & -8 & -8 \\
\cline { 2 - 6 } & pval & 0.00 & 0.10 & 0.00 & 0.10 \\
\hline \multirow{2}{*}{ miR-494 } & $\mathrm{FC}$ & 3 & 2 & -6 & -10 \\
\cline { 2 - 6 } & pval & 0.08 & 0.14 & 0.08 & 0.14 \\
\hline \multirow{2}{*}{ miR-495 } & $\mathrm{FC}$ & 7 & 3 & -5 & -12 \\
\cline { 2 - 6 } & pval & 0.00 & 0.09 & 0.00 & 0.09 \\
\hline \multirow{2}{*}{ miR-539 } & $\mathrm{FC}$ & 16 & 5 & -17 & -51 \\
\cline { 2 - 6 } & pval & 0.03 & 0.08 & 0.03 & 0.08 \\
\hline
\end{tabular}

\section{C}

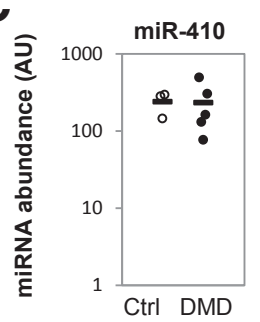

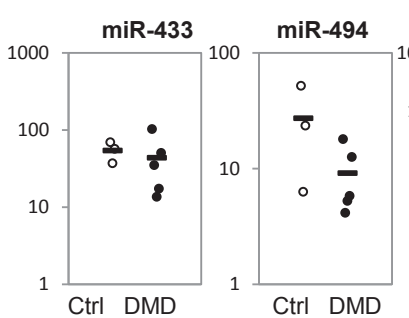

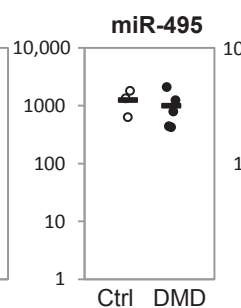

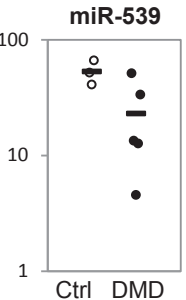

D

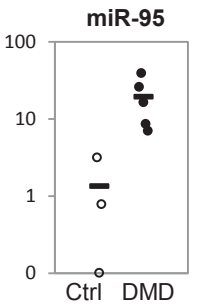

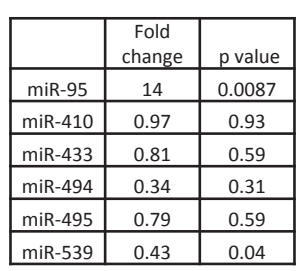

Figure 5 DLK1-DI03 locus miRNAs expression in $m d x$ mouse and Duchenne muscular dystrophy (DMD) patients. A: DLK1-DI03 miRNAs were quantified in the serum in $m d x$ (black circles) and control mice (open circles). Expression levels were normalized to miR-16 and are expressed in abundance AU. B: FCs and $P$ values are given. C: Quantification of DLK1-DIO3 locus miRNAs and miR-95 in serum of DMD patients (black circles) and age-matched controls (open circles). Expression levels were normalized to miR-16 and are expressed in abundance AU. Each dot represents one donor. Mean miRNA abundance values are represented by a black horizontal line. D: FCs and $P$ values are given. Wilcoxon rank sum test are indicated. Median, mean, and SD of all tested miRNAs for the different mouse and human groups are given in Supplemental Table S9. Each dot $(n=3)$ represents a pool of three mice (serum mix) of the indicated strain and age (A); $n=5$ DMD patients (C); $n=3$ age-matched controls (C). $P<0.05$ (B). AU, arbitrary unit; Bl6, C57bl/6 mice; Ctrl, control; $\mathrm{m}$, minutes; pval, $P$ value.

group of the previously identified dystromiRs, including miR-1, miR-133a, miR-133b, miR-206, miR-378, and miR$378^{*}$, we can add miR-95, a miRNA expressed in dogs and humans, but not in rodents, whose gene is located in an intron of the Ablim-2 or ABLIM-2 gene, respectively. This gene encodes an F-actin binding protein that is expressed preferentially in the muscular and nervous systems. ${ }^{37}$ However, unlike miR-1, miR-133a, miR-206, and miR-378, which are already highly elevated in the serum in GRMD dogs at birth (Figure 2) and are significantly up-regulated at all latter ages, miR-95 is dysregulated between the ages of 1.5 and 7 months. How can we explain the differences in the kinetics of serum dytromiRs up-regulation? The release of dystromiRs into the serum might be suspected to be caused by a passive leakiness of damaged myofibers, which in turn may imply similar dysregulation kinetics of the different dystromiRs (serum level change over time and disease stage in dystrophic subjects). Yet, not all myofiber-enriched miRNAs are upregulated in the serum of dystrophic subjects. For example the Let-7 family miRNAs, some of which are highly expressed in muscle, ${ }^{43}$ are not dysregulated in the serum of dystrophic mice ${ }^{22}$ and dogs (present study). Thus, the release of myofiber-enriched miRNAs into the circulation is not a merely passive process, and identical dysregulation kinetics of the dystromiRs is not expected. The type and nature of the molecular processes that control miRNAs release from damaged and necrotic myofibers into the circulation are yet to be studied.

Another newly identified group of dysregulated miRNAs in GRMD dog serum resides in the imprinted DLK1-DIO3 genomic locus. The dysregulation of this miRNA mega-cluster and its involvement in pathogenesis have recently been reviewed. ${ }^{44}$ In skeletal muscle, this entire miRNA cluster was found to be regulated by the MEF2A transcription factor and up-regulated in regenerating muscles ${ }^{41}$ suggesting its involvement in muscle pathophysiology. We have identified higher serum content in GRMD dogs of a large number of miRNAs of this cluster, of which 40 were statistically significant (Figure 1 and Supplemental Table S2). Of these, the up-regulation of five miRNAs was validated by an individual 
A

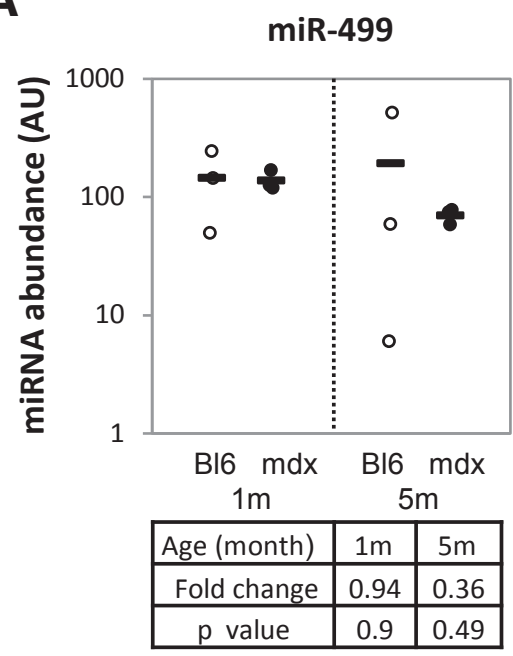

miR-208a
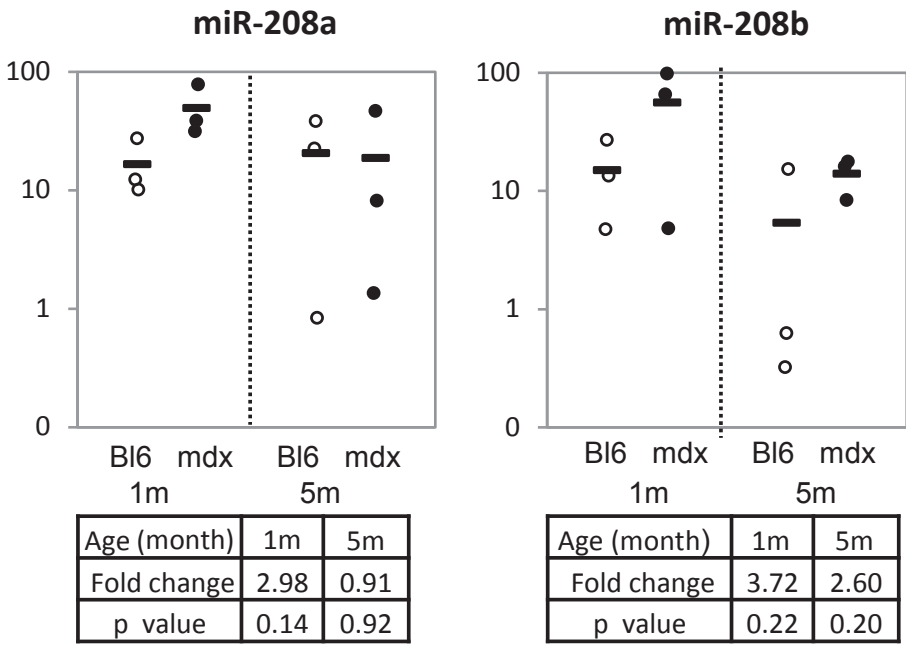

B
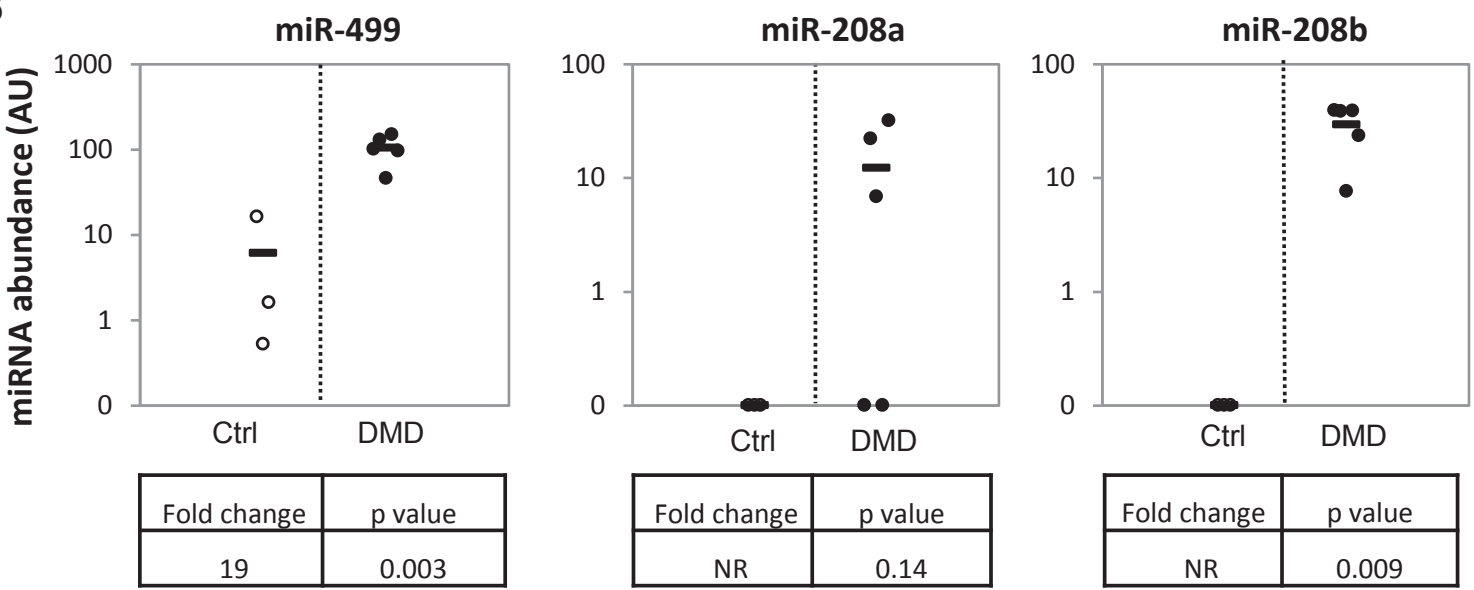

Figure 6 Cardiac-enriched miRNAs are dysregulated in Duchenne muscular dystrophy (DMD) patients but not in mdx mice. A: Cardiac-enriched miRNAs were quantified in the serum in $m d x$ (black circles) and control mice (open circles). Expression in AUs is normalized to miR-16. FCs and $P$ values (Wilcoxon rank sum test) are indicated. B: Cardiac-enriched miRNAs were quantified in the serum of DMD patients (black circles) and agematched controls (open circles). Expression in AUs is normalized to miR-16. Each dot represents one donor. Mean values are represented by a black horizontal line. Fold changes and $P$ values (Wilcoxon rank sum test) are indicated below. Each dot $(n=3)$ represents a pool (serum mix) of three mice of the indicated strains and ages (A). $n=5$ DMD patients (B); $n=3$ age-matched controls (B); AU, arbitrary unit; BI6, C57bl/6 mice; Ctrl, control; $\mathrm{NR}$, nonrelevant (no expression in controls).

RT-qPCR, with a highly correlating expression pattern, suggesting their co-dysregulation in GRMD dog serum. Expression levels in both dystrophic and control subjects were age dependent in both mouse and dog, suggesting in addition to a pathology-dependent expression pattern a developmentaldependence expression, which is expected from imprinting loci. Interestingly, a contradictory trend was observed for miR539, identified as up-regulated in GRMD (Supplemental Table $\mathrm{S} 2$ ), confirmed as up-regulated in the $m d x$ mouse (Figure $5 \mathrm{~A}$ ), but significantly down-regulated in human DMD patients (Figure 5B). As mentioned above, the expression of the DLK1DIO3 miRNAs seemed to be affected by both the muscle regeneration transcription program and an age-dependent developmental stage, a process that is different between the studied species and can explain this observed discrepancy. Although the biomarker utility of the serum DLK1-DIO3 cluster miRNAs in DMD patients is yet not clear, the identification of their dysregulation in muscular dystrophy animal models should motivate further investigations of their role in muscle pathophysiology.

DMD is a multisystem disorder, affecting principally both skeletal and cardiac muscles. Therapeutic approaches may preferentially target skeletal or cardiac muscles. Antisense oligonucleotide-mediated exon skipping, for example, induces higher dystrophin restoration in skeletal muscle than in cardiac muscle in the $m d x$ mouse $^{45}$ and in human patients, ${ }^{46}$ emphasizing the necessity of monitoring both type of tissues independently. One clear predicted advantage of the circulating miRNA biomarker approach for disease monitoring is the ability to monitor simultaneously distinct affected tissues. However, to our knowledge this type of application has not yet been described. Ongoing 


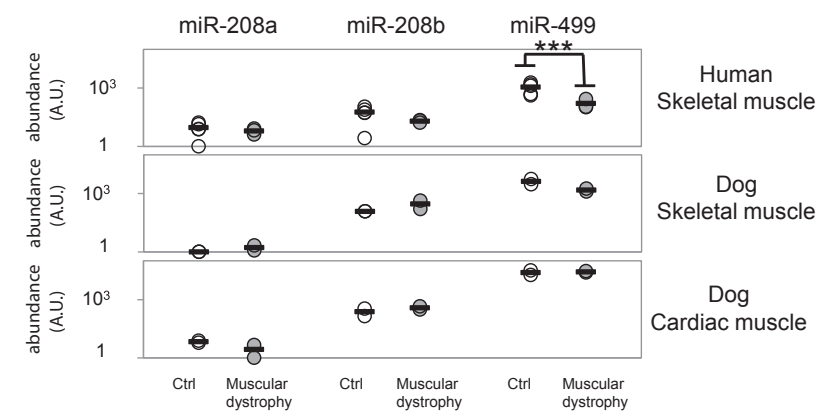

Figure 7 Cardiac-enriched miRNA expression in skeletal and cardiac muscle tissues. miRNAs were quantified in skeletal muscles in dystrophic (gray circles) and control (open circles) in dog and human and in cardiac muscle in dogs. Skeletal muscle biopsies were derived from the parvalateral muscle, dorsal muscle, and the ulnar muscle (all dog biopsies, 6- to 7-month-old GRMD and controls). Expression in AUs is normalized to miR16. $n=2$ parvalateral muscle biopsies from DMD patients; $n=3$ parvalateral muscle biopsies from human controls; $n=1$ dorsal muscle biopsy from DMD patients. ${ }^{* *} P<0.001$. AU, arbitrary unit; Ctrl, control.

work on circulating miRNA biomarkers for both skeletal and cardiac muscle pathology in DMD will investigate this possibility.

Indeed in the present study we identified in the serum of GRMD dogs the dysregulation of a group of cardiac-enriched miRNAs, which are miR-499, miR-208a, and miR-208b. The utility of these miRNAs as monitors of cardiac clinical situation was studied, in comparison with cardiac Tpn, in a GRMD dog cohort that included subjects with various degrees of myocardial contractility. We found no simple correlation between the serum levels of cardiac miRNAs and cardiac Tpn and the degree of heart functional performance (including FS and several other indices). miR-499 and miR$208 \mathrm{~b}$ were reported to be expressed in the mouse not only in the heart but also in skeletal muscle slow twitch fibers, whereas miR-208a was reported to be heart specific. ${ }^{47}$ With the use of a highly sensitive PCR assay, we confirmed the expression of miR-208b and miR-499 in dog and human skeletal muscle and surprisingly also observed the expression of miR-208a (Figure 7). Further, in GRMD dogs, the expression of miR-208a in the heart was only 1.6-fold higher than in skeletal muscle. Given that the total mass of skeletal muscle is several fold higher than that of heart muscle, the relative contribution of circulating miR-208a originating from skeletal muscles may exceed the contribution of heart muscle in GRMD dogs. Consistently, serum expression level of miR-208a was positively correlated with the expression of the respective cardiac and skeletal muscle damage biomarkers, cardiac Tpn and muscle CK, whereas miR-208a and miR-499 expression correlated only with expression of muscle CK (Figure 4C). Detection of miR-208a expression in human skeletal muscle has been reported by others. ${ }^{48,49}$ Collectively, this suggests that in dog and human, expression of miR-208a is not limited to the heart. Thus, serum dysregulation of miR-208b, miR-499, and miR-208a can be caused by both skeletal and cardiac muscle pathology in dystrophic subjects (dog and human). Yet, serum expression of three cardiac-enriched miRNAs positively correlated with the skeletal muscle damage biomarker $\mathrm{CK}$, whereas the cardiac muscle damage biomarker cardiac Tpn correlated slightly positively only with miR-208a.

Some of the GRMD dogs, including subjects with low value of FS percentage, were almost or completely negative for serum miR-208a and for cardiac Tpn (Figure 4A), supporting a notion that some GRMD dogs with cardiomyopathy are negative, occasionally at least, for cardiomyopathy-related biomarker(s). Moreover, we found that serum levels of miR-208a, but not of miR-208b and miR-499, were strongly fluctuating over time in GRMD dog serum (Figure 4B). A possible explanation is that successive temporal episodes of cardiomyocyte degeneration in GRMD dogs result in release of cardiac Tpn and miR-208a into the serum, which is followed by transitory remission periods and clearance of these biomarkers from the circulation. However, further experimental evidence is needed to validate this interpretation.

Taken together, these data suggest that cardiac miRNA serum dysregulation in GRMD dogs is influenced by cardiac damage but does not correlate with cardiac phenotype, possibly because none of these miRNAs are expressed specifically in the heart, and serum expression of miR-208a is fluctuating over time, thus limiting the utility of cardiac miRNAs for the monitoring of cardiac pathology in GRMD.

It would be interesting to investigate the utility of earlyage detection of serum miR-208a in GRMD as a predictive biomarker for the precocity and severity of cardiomyopathy with aging. Unfortunately, this investigation could not be accomplished in the present study because no early serum samples were available from the GRMD dogs that underwent cardiac functional assessment. This important question will have to be addressed in future studies in GRMD dogs and DMD patients.

\section{Conclusion}

The GRMD dog model was proved to be useful for biomarker discovery. miRNA HTS was confirmed as a robust method for serum miRNA profiling and for identifying dysregulation. We identified in the serum in GRMD dogs three distinct groups of dysregulated miRNAs: the dystromiRs, the DLK1-DIO3 miRNAs, and the cardiacenriched miRNAs. Their dysregulation was characterized in a detailed large-scale longitudinal study. No correlations were identified in GRMD dogs between the serum level of cardiac miRNAs and cardiac functional assessment. The dysregulation of the dystromiR miR-95, of the DLK1-DIO3 miRNA miR-539, and of the cardiac-enriched miRNAs was confirmed in a small DMD patient cohort. Profiling circulating miRNAs in ongoing and future preclinical and clinical studies in DMD patients and animal models will allow further investigation of the biomarker potential of these newly identified candidates. 


\section{Acknowledgments}

We thank the Centre d'Elevage du Domaine des Souches for providing some of the serum samples and breeding the French GRMD colony; Dr Benoît Rannou and Edwige Rousseilère (Laboratoire de biologie médicale, VetAgroSup Lyon) for the dosages of TpnI and their expert support; the Cardiology unit and INSERM U955 (Ecole Nationale Vétérinaire d'Alfort) for performing the echocardiographic evaluation of GRMD dogs; Myobank, the tissue bank of the AFM, and the Myology Institute (Paris, France) for providing DMD skeletal muscle tissues; and Integragen (Evry, France), our partner, for high-throughput sequencing.

L.J.-L. designed and performed experiments, analyzed results, and wrote the paper; J.L., S.K., J.B., and F.A. performed experiments; C.L.G., I.B., L.S., S.B., and T.V. contributed material and analyzed results; and D.I. designed experiments, analyzed results, and wrote the paper.

\section{Supplemental Data}

Supplemental material for this article can be found at http://dx.doi.org/10.1016/j.ajpath.2014.07.021.

\section{References}

1. Passamano L, Taglia A, Palladino A, Viggiano E, D'Ambrosio P, Scutifero M, Rosaria Cecio M, Torre V, De Luca F, Picillo E, Paciello O, Piluso G, Nigro G, Politano L: Improvement of survival in Duchenne Muscular Dystrophy: retrospective analysis of 835 patients. Acta Myol 2012, 31:121-125

2. Kieny P, Chollet S, Delalande P, Le Fort M, Magot A, Pereon Y, Perrouin Verbe B: Evolution of life expectancy of patients with Duchenne muscular dystrophy at AFM Yolaine de Kepper centre between 1981 and 2011. Ann Phys Rehabil Med 2013, 56:443-454

3. Banks GB, Chamberlain JS: The value of mammalian models for duchenne muscular dystrophy in developing therapeutic strategies. Curr Top Dev Biol 2008, 84:431-453

4. Kornegay JN, Bogan JR, Bogan DJ, Childers MK, Li J, Nghiem P, Detwiler DA, Larsen CA, Grange RW, Bhavaraju-Sanka RK, Tou S, Keene BP, Howard JF Jr, Wang J, Fan Z, Schatzberg SJ, Styner MA, Flanigan KM, Xiao X, Hoffman EP: Canine models of Duchenne muscular dystrophy and their use in therapeutic strategies. Mamm Genome 2012, 23:85-108

5. Emery AE: The muscular dystrophies. Lancet 2002, 359:687-695

6. Gasper MC, Gilchrist JM: Creatine kinase: a review of its use in the diagnosis of muscle disease. Med Health R I 2005, 88:398. 400-404

7. Rouger K, Larcher T, Dubreil L, Deschamps JY, Le Guiner C, Jouvion G, Delorme B, Lieubeau B, Carlus M, Fornasari B, Theret M, Orlando P, Ledevin M, Zuber C, Leroux I, Deleau S, Guigand L, Testault I, Le Rumeur E, Fiszman M, Chérel Y: Systemic delivery of allogenic muscle stem cells induces long-term muscle repair and clinical efficacy in duchenne muscular dystrophy dogs. Am J Pathol 2011, 179:2501-2518

8. Barthélémy I, Uriarte A, Drougard C, Unterfinger Y, Thibaud JL, Blot S: Effects of an immunosuppressive treatment in the GRMD dog model of Duchenne muscular dystrophy. PLoS One 2012, 7:e48478

9. Wood MJ: To skip or not to skip: that is the question for duchenne muscular dystrophy. Mol Ther 2013, 21:2131-2132

10. Hoffman EP, McNally EM: Exon-skipping therapy: a roadblock, detour, or bump in the road? Sci Transl Med 2014, 6:230fs14
11. Wilton SD, Fletcher S, Flanigan KM: Dystrophin as a therapeutic biomarker: are we ignoring data from the past? Neuromuscul Disord 2014, 24:463-466

12. Hoffman EP, Connor EM: Orphan drug development in muscular dystrophy: update on two large clinical trials of dystrophin rescue therapies. Discov Med 2013, 16:233-239

13. Kelley WE, Januzzi JL, Christenson RH: Increases of cardiac troponin in conditions other than acute coronary syndrome and heart failure. Clin Chem 2009, 55:2098-2112

14. Matsumura T, Saito T, Fujimura H, Shinno S: Cardiac troponin I for accurate evaluation of cardiac status in myopathic patients. Brain Dev 2007, 29:496-501

15. Castro-Gago M, Gómez-Lado C, Eirís-Puñal J: Cardiac troponin I for accurate evaluation of cardiac status in myopathic patients (letter to the editor). BrainDev 2009, 31:184

16. Ergul Y, Ekici B, Nisli K, Tatli B, Binboga F, Acar G, Ozmen M, Omeroglu RE: Evaluation of the North Star Ambulatory Assessment scale and cardiac abnormalities in ambulant boys with Duchenne muscular dystrophy. J Paediatr Child Health 2012, 48: 610-616

17. Schade van Westrum S, Dekker L, de Haan R, Endert E, Ginjaar I, de Visser M, van der Kooi A: Brain natriuretic peptide is not predictive of dilated cardiomyopathy in Becker and Duchenne muscular dystrophy patients and carriers. BMC Neurol 2013, 13:88

18. Weber JA, Baxter DH, Zhang S, Huang DY, Huang KH, Lee MJ, Galas DJ, Wang K: The microRNA spectrum in 12 body fluids. Clin Chem 2010, 56:1733-1741

19. Mitchell PS, Parkin RK, Kroh EM, Fritz BR, Wyman SK, PogosovaAgadjanyan EL, Peterson A, Noteboom J, O'Briant KC, Allen A, Lin DW, Urban N, Drescher CW, Knudsen BS, Stirewalt DL, Gentleman R, Vessella RL, Nelson PS, Martin DB, Tewari M: Circulating microRNAs as stable blood-based markers for cancer detection. Proc Natl Acad Sci U S A 2008, 105:10513-10518

20. Chen X, Ba Y, Ma L, Cai X, Yin Y, Wang K, Guo J, Zhang Y, Chen J, Guo X, Li Q, Li X, Wang W, Zhang Y, Wang J, Jiang X, Xiang Y, Xu C, Zheng P, Zhang J, Li R, Zhang H, Shang X, Gong T, Ning G, Wang J, Zen K, Zhang J, Zhang CY: Characterization of microRNAs in serum: a novel class of biomarkers for diagnosis of cancer and other diseases. Cell Res 2008, 18:997-1006

21. Cortez MA, Bueso-Ramos C, Ferdin J, Lopez-Berestein G, Sood AK, Calin GA: MicroRNAs in body fluids-the mix of hormones and biomarkers. Nat Rev Clin Oncol 2011, 8:467-477

22. Vignier N, Amor F, Fogel P, Duvallet A, Poupiot J, Charrier S, Arock M, Montus M, Nelson I, Richard I, Carrier L, Servais L, Voit T, Bonne G, Israeli D: Distinctive serum miRNA profile in mouse models of striated muscular pathologies. PLoS One 2013, 8:e55281

23. Cacchiarelli D, Legnini I, Martone J, Cazzella V, D'Amico A, Bertini E, Bozzoni I: miRNAs as serum biomarkers for Duchenne muscular dystrophy. EMBO Mol Med 2011, 3:258-265

24. Mizuno H, Nakamura A, Aoki Y, Ito N, Kishi S, Yamamoto K, Sekiguchi M, Takeda S, Hashido K: Identification of muscle-specific microRNAs in serum of muscular dystrophy animal models: promising novel blood-based markers for muscular dystrophy. PLoS One 2011, 6:e18388

25. Roberts TC, Blomberg KE, McClorey G, Andaloussi SE, Godfrey C, Betts C, Coursindel T, Gait MJ, Smith CI, Wood MJ: Expression analysis in multiple muscle groups and serum reveals complexity in the microRNA transcriptome of the mdx mouse with implications for therapy. Mol Ther Nucleic Acids 2012, 1:e39

26. Zaharieva IT, Calissano M, Scoto M, Preston M, Cirak S, Feng L, Collins J, Kole R, Guglieri M, Straub V, Bushby K, Ferlini A, Morgan JE, Muntoni F: Dystromirs as serum biomarkers for monitoring the disease severity in Duchenne muscular dystrophy. PLoS One 2013, 8:e80263

27. Duguez S, Duddy W, Johnston H, Laine J, Le Bihan MC, Brown KJ, Bigot A, Hathout Y, Butler-Browne G, Partridge T: Dystrophin deficiency leads to disturbance of LAMP1-vesicle-associated protein secretion. Cell Mol Life Sci 2013, 70:2159-2174 
28. Le Bihan MC, Bigot A, Jensen SS, Dennis JL, RogowskaWrzesinska A, Laine J, Gache V, Furling D, Jensen ON, Voit T, Mouly V, Coulton GR, Butler-Browne G: In-depth analysis of the secretome identifies three major independent secretory pathways in differentiating human myoblasts. J Proteomics 2012, 77:344-356

29. Creemers EE, Tijsen AJ, Pinto YM: Circulating microRNAs: novel biomarkers and extracellular communicators in cardiovascular disease? Circ Res 2012, 110:483-495

30. Corsten MF, Dennert R, Jochems S, Kuznetsova T, Devaux Y, Hofstra L, Wagner DR, Staessen JA, Heymans S, Schroen B: Circulating MicroRNA-208b and MicroRNA-499 reflect myocardial damage in cardiovascular disease. Circ Cardiovasc Genet 2010, 3:499-506

31. Oliveira-Carvalho V, Carvalho VO, Bocchi EA: The emerging role of miR-208a in the heart. DNA Cell Biol 2013, 32:8-12

32. Wang GK, Zhu JQ, Zhang JT, Li Q, Li Y, He J, Qin YW, Jing Q: Circulating microRNA: a novel potential biomarker for early diagnosis of acute myocardial infarction in humans. Eur Heart J 2010, 31: 659-666

33. Chetboul V, Athanassiadis N, Carlos C, Nicolle A, Zilberstein L, Pouchelon JL, Lefebvre HP, Concordet D: Assessment of repeatability, reproducibility, and effect of anesthesia on determination of radial and longitudinal left ventricular velocities via tissue Doppler imaging in dogs. Am J Vet Res 2004, 65:909-915

34. Si JB, Carzorla O, Blot S, Blanchard-Gulton N, Ait Mou Y, Barthélémy I, Sambin L, Sampedrano CC, Gouni V, Unterfinger Y, Aguilar P, Thibaud JL, Bizé A, Pouchelon JL, Dabiré H, Ghaleh B, Berdeaux A, Chetboul V, Lacampagne A, Hittinger L: Bradykinin restores left ventricular function, sarcomeric protein phosphorylation, and e/nNOS levels in dogs with Duchenne muscular dystrophy cardiomyopathy. Cardiovasc Res 2012, 95:86-96

35. Vigneault F, Ter-Ovanesyan D, Alon S, Eminaga S, Christodoulou DC, Seidman JG, Eisenberg E, Church GM: Highthroughput multiplex sequencing of miRNA. Curr Protoc Hum Genet 2012. Chapter 11:Unit 11.12.1-10

36. Hackenberg M, Rodríguez-Ezpeleta N, Aransay AM: miRanalyzer: an update on the detection and analysis of microRNAs in high-throughput sequencing experiments. Nucleic Acids Res 2011, 39:W132-W138

37. Barrientos T, Frank D, Kuwahara K, Bezprozvannaya S, Pipes GC, Bassel-Duby R, Richardson JA, Katus HA, Olson EN, Frey N: Two novel members of the ABLIM protein family, ABLIM-2 and -3 , associate with STARS and directly bind F-actin. J Biol Chem 2007, 282:8393-8403

38. Edwards CA, Mungall AJ, Matthews L, Ryder E, Gray DJ, Pask AJ, Shaw G, Graves JA, Rogers J; SAVOIR consortium, Dunham I,
Renfree MB, Ferguson-Smith AC: The evolution of the DLK1-DIO3 imprinted domain in mammals. PLoS Biol 2008, 6:e135

39. Byrne K, Colgrave ML, Vuocolo T, Pearson R, Bidwell CA, Cockett NE, Lynn DJ, Fleming-Waddell JN, Tellam RL: The imprinted retrotransposon-like gene PEG11 (RTL1) is expressed as a full-length protein in skeletal muscle from Callipyge sheep. PLoS One 2010, 5:e8638

40. Fleming-Waddell JN, Olbricht GR, Taxis TM, White JD, Vuocolo T, Craig BA, Tellam RL, Neary MK, Cockett NE, Bidwell CA: Effect of DLK1 and RTL1 but not MEG3 or MEG8 on muscle gene expression in Callipyge lambs. PLoS One 2009, 4:e7399

41. Snyder CM, Rice AL, Estrella NL, Held A, Kandarian SC, Naya FJ: MEF2A regulates the Gt12-Dio3 microRNA mega-cluster to modulate WNT signaling in skeletal muscle regeneration. Development 2013, 140:31-42

42. Li C, Fang Z, Jiang T, Zhang Q, Liu C, Zhang C, Xiang Y: Serum microRNAs profile from genome-wide serves as a fingerprint for diagnosis of acute myocardial infarction and angina pectoris. BMC Med Genomics 2013, 6:16

43. Hou X, Tang Z, Liu H, Wang N, Ju H, Li K: Discovery of MicroRNAs associated with myogenesis by deep sequencing of serial developmental skeletal muscles in pigs. PLoS One 2012, 7:e52123

44. Benetatos L, Hatzimichael E, Londin E, Vartholomatos G, Loher P, Rigoutsos I, Briasoulis E: The microRNAs within the DLK1-DIO3 genomic region: involvement in disease pathogenesis. Cell Mol Life Sci 2013, 70:795-814

45. Heemskerk HA, de Winter CL, de Kimpe SJ, van Kuik-Romeijn P, Heuvelmans N, Platenburg GJ, van Ommen GJ, van Deutekom JC, Aartsma-Rus A: In vivo comparison of 2'-O-methyl phosphorothioate and morpholino antisense oligonucleotides for Duchenne muscular dystrophy exon skipping. J Gene Med 2009, 11:257-266

46. Muntoni F, Wood MJ: Targeting RNA to treat neuromuscular disease. Nat Rev Drug Discov 2011, 10:621-637

47. van Rooij E, Quiat D, Johnson BA, Sutherland LB, Qi X, Richardson JA, Kelm RJ Jr, Olson EN: A family of microRNAs encoded by myosin genes governs myosin expression and muscle performance. Dev Cell 2009, 17:662-673

48. Davidsen PK, Gallagher IJ, Hartman JW, Tarnopolsky MA, Dela F, Helge JW, Timmons JA, Phillips SM: High responders to resistance exercise training demonstrate differential regulation of skeletal muscle microRNA expression. J Appl Physiol (1985) 2011, 110:309-317

49. Greco S, Perfetti A, Fasanaro P, Cardani R, Capogrossi MC, Meola G, Martelli F: Deregulated microRNAs in myotonic dystrophy type 2. PLoS One 2012, 7:e39732 